\title{
Phytodiversite Et Pharmacopée Traditionnelle De La Communauté Kaamba De Madingou (Bouenza - Congo)
}

\author{
Victor Kimpouni \\ Marie-Yvette Lenga-Sacadura \\ Josérald Chaîph Mamboueni
}

École normale supérieure, Université Marien Ngouabi, Brazzaville, Congo

\section{Elie Nsika Mikoko}

Faculté des sciences et technique, Université Marien Ngouabi,

Brazzaville, Congo

Doi: 10.19044/esj.2018.v14n3p191 URL:http://dx.doi.org/10.19044/esj.2018.v14n3p191

\begin{abstract}
The ethnobotanical study carried out at Madingou ( $\left.4^{\circ} 16^{\prime} \mathrm{S}, 13^{\circ} 33^{\prime} \mathrm{E}\right)$ focuses on medicinal plants of the savannah zone. The survey used personal interviews and focus groups. Informants consisted of the inhabitants, whose age varies from 18 to 60 years or more. A total of 80 plant species were cited in the traditional pharmacopoeia. These plants are used in 66 recipes for treating 43 different diseases and symptoms, grouped in 11 major affiliations following the disease and symptoms sphere. Infectious and parasitic diseases is the most important group (23.25\%), followed by the ill-defined symptoms and signs (13.95\%) and the digestive system (11.63\%). Five modes of administration were noted. The most preferred being the oral voice $(38.75 \%)$. Among the 7 types of plant parts cited, leaves (65\%) are preponderant. 8 modes of preparation were cited, the decoction $(23.75 \%)$ being the most common. Of the 80 species listed, Allium sativum, Persea americana, Solanum nigrum, are among the medicinal plants most commonly used in Africa. Of the remaining species, 9 have been the subject of phytochemical studies all over the world. Ethnobotanical data show that a number of taxa, the medicinal properties of which are proved elsewhere, exist in the region and are not exploited, such as Millettia laurentii. Several species inventoried cover more than one aspect of indigenous knowledge, they are referred to as multipurpose plants and therefore the most vulnerable of the anthropic influences.
\end{abstract}

Keywords: Madingou; ethnobotany; diseases; recipes; symptoms; traditional uses; phytotherapic 


\section{Résumé}

L'étude ethnobotanique menée à Madingou ( $\left.4^{\circ} 16^{\prime} \mathrm{S} ; 13^{\circ} 33^{\prime} \mathrm{E}\right)$, porte sur la pharmacopée traditionnelle des peuples de savanes. L'enquête se focalise sur les interviews personnalisées et les «focus group ». En l'absence de tradithérapeutes professionnels, nous nous contentons des savoirs endogènes propres aux habitants, dont l'âge varie de 18 à 60 ans voire plus. L'inventaire floristique ressort 80 espèces associées à la pharmacopée traditionnelle. L'aspect phytothérapeutique regroupe 66 recettes pour 43 maladies et symptômes divers. Suivant la sphère de la maladie et des symptômes, 11 grandes affiliations sont relevées. Cette classification montre une dominance des maladies infectieuses et parasitaires $(23,25 \%)$ devant les symptômes et signes mal définis (13,95\%) et les maladies de l'appareil digestif (11,63\%). Cinq modes d'administration ont été relevés. La voie per os $(63 \%)$ est le plus prôné. On compte, 9 types organes utilisés pour la préparation des recettes. Les feuilles $(65 \%)$ sont les organes les plus prépondérantes. Un total de 8 modes de préparations a été recensé. La décoction $(47,5 \%)$ est le mode de préparation le plus utilisé Des 80 espèces recensées, Allium sativum, Persea americana, Solanum nigrum, figurent parmi les plantes les plus usitées en Afrique. Neuf espèces ont fait l'objet d'études phytochimiques de par le monde. Les données ethnobotaniques montrent qu'un nombre de taxons, aux vertus médicinales prouvées ailleurs, existent dans la contrée sans être exploités, à l'instar de Millettia laurentii. Plusieurs espèces inventoriées couvrent plus d'un aspect des savoirs endogènes; elles sont qualifiées de plantes à usages multiples et donc les plus vulnérables aux actions anthropiques.

Mots clés : Madingou; ethnobotanique ; maladies ; recettes ; symptômes ; usages traditionnels; phytothérapie

\section{Introduction}

Depuis la préhistoire, l'être humain recherche dans son environnement les moyens d'améliorer son bien-être social et traiter ses blessures (Sofowora, 1996). Les procédés qu'il se donne, sont l'exploitation des vertus de la flore, de la faune et minérale, mise en évidence par ses aïeuls (Pousset, 1998 ; Dupius 2011). Parmi ceux-ci, nous soulignons les traitements médicinaux à base des plantes (pharmacopée traditionnelle). Partout dans le monde, l'intérêt pour la médecine traditionnelle s'accroit constamment ; à cause, des coûts prohibitifs de la médecine moderne qui poussent les populations démunies vers (i) l'automédication (utilisation des médicaments de la rue) qui est une pratique très dangereuse et/ou (ii) la phytothérapie. En zones rurales, le manque d'instituts médicaux à proximité, est l'une des causes de la dépendance de ces 
populations à la pharmacopée traditionnelle. Cette pharmacopée traditionnelle permet aussi la découverte et la synthèse des nouvelles molécules dont se sert la médecine moderne (Farsworth et al., 1986 ; Verpoorte, 2000 ; Wezel, 2002 ; Sanogo, 2006). Comparativement aux avancées de la pharmacopée traditionnelle chinoise ou indienne, en Afrique subsaharienne notamment, la pratique de cette médecine nécessite une meilleure prise en compte dans le système de santé. Comme le montre l'étude de Farsworth et al. (1986), 119 substances chimiques obtenues à partir de 91 espèces végétales, étaient utilisées comme des médicaments primordiaux dans 62 classes thérapeutiques. En 1995, des 25 produits pharmaceutiques les mieux vendus dans le monde, 12 étaient d'origine naturelle (Baker et al., 1995). En 1999, environ 139000 métabolites secondaires ont été recensés dans le Dictionnaire des produits naturels et environ 4000 nouvelles molécules sont recensées chaque année (Verpoorte, 2000). En effet, on observe que beaucoup d'études ont été menées dans la société africaine, mais elles restent très parcellaires, ce qui rend difficile l'éclosion de la phytothérapie. Dans plusieurs régions reculées, les plantes médicinales constituent pour les guérisseurs traditionnels une ressource à la résolution des problèmes de santé. Les données de la littérature font remarquer que $90 \%$ de la population de ces contrées font recours à ces méthodes (Sofowora, 1996 ; Sanogo, 2006 ; Dupius, 2011).

Le Congo, localisé en Afrique centrale, couvre une superficie de $342000 \mathrm{Km}^{2}$ dont 35 à 40\% sont couverts de savane (Vennetier, 1977). Elle recouvre les plateaux Téké, la vallée du Niari, les Cataractes et la plaine côtière (UICN, 1996). Les travaux d'ethnobotaniques connus sont généralement globalisants et ne ressortent pas les spécificités culturelles de chaque groupe ethnique, socle des savoirs endogènes. D'où, l'intérêt de cette étude ethnobotanique menée dans le district de Madingou au sein du groupe ethnique Kaamba, afin de combler un hiatus sur les connaissances endogènes en général et des peuples de savane en particulier.

Aujourd'hui, ces savoirs transmis oralement de génération en génération, connaissent un déclin découlant de l'émergence de la société vers le modernisme. Parmi les maux qui minent le transfert des savoirs, citons : (i) l'exode rural entraine un déplacement de masse, notamment des jeunes, du monde rural vers la ville où ils espèrent améliorer leur bien-être ; (ii) Le vieillissement de la population couplé à l'exode rural des jeunes accélère cette décadence ; (iii) l'urbanisation plus ou moins anarchique, dont les travaux menés sans études d'impact environnemental et social, conduit inéluctablement à la perte de la biodiversité suite à la destruction des niches écologiques ; affecte par conséquent la perpétuation des savoirs être, faire et vivre ; (iv) le feu de brousse, par son effet sélectif, est une cause de la perte des plantes utiles, ce qui est à l'origine de l'abandon de certaines recettes (Frontier et al., 2008). 
Ces perturbations (déforestation et exploitation industrielle du bois, urbanisation anarchique, feu de brousse) affectent le fonctionnement et les équilibres vitaux des écosystèmes. Bergonzini (2004) et Riera \& Alexandre (2004) mentionnent qu'environ 34000 espèces végétales sont sous la menace d'une disparition à plus ou moins court terme. Ces faits loin d'être anodins pénalisent les adeptes de la pharmacopée traditionnelle. C'est ainsi que ces pratiques, socle socioculturel au service de la communauté, sont progressivement oubliées. En les couplant à l'exode rural, citons «... en Afrique, un vieillard qui meurt est une bibliothèque qui brûle » (Betti et al., 2013a, 2013b ; Raponda-Walker \& Sillans, 1961).

Les écosystèmes du Congo soumis aux contraintes climatiques (changements globaux) en plus de l'action anthropique sont très vulnérables. Leur biodiversité très partiellement connue, la valorisation et la durabilité desdits milieux s'imposent en général et particulièrement les savanes, comme le soulignent Gartlan (1989) et Bergonzini (2004). Les écosystèmes tropicaux très riches et diversifiés (Puig, 2001), connaissent une régression constante des superficies dont les effets sont une diminution des ressources floristiques, une érosion des taxons et des gènes (Puig, 2001 ; Slayback, 2003 ; Bergonzini, 2004). Les savoirs endogènes liés à la phytothérapie traditionnelle, justifient la conservation des ressources phytogénétiques, pour que soit perpétuer ces valeurs socioculturelles et amender la médecine, afin du développement de la société.

Au regard de la répartition de la population, les zones de savanes sont les plus densifiées (Vennetier, 1977) mais, les travaux sur les savoirs endogènes au Congo, se cristallisent presque toujours dans les zones forestières (Adjanohoun et al., 1988 ; Bouquet,1969 ; Makany, 1976). Ceci porte à croire que les savanes ne regorgent pas de plantes médicinales et les populations qui y vivent n'ont aucune emprise sur la biodiversité à portée de la main, pour la satisfaction des besoins de santé primaire (Grenand \& Prévost, 1994 ; Grenand et al., 1987).

\section{Objectif}

Ce travail sur les savoirs endogènes des Kaamba de Madingou, notamment la pharmacopée traditionnelle, s'inscrit dans le cadre général de l'amélioration des connaissances sur l'homme et son environnement. En se focalisant sur la phytothérapie traditionnelle, son centre d'intérêt est : (i) l'inventaire des plantes médicinales ; (ii) la caractérisation de la pharmacopée locale.

\section{Hypothèse d'étude}

Les hypothèses de travail sont: (i) la flore des savanes présente une phytodiversité exploitée en pharmacopée traditionnelle par la communauté 
Kaamba ; (ii) cette étude met en exergue les spécificités des savoirs endogènes du groupe ethnique Kaamba ; (iii) l'exode rural et la disparition des personnes âgées, sont deux paramètres qui influencent négativement sur la transmission des savoirs empiriques.

\section{Matériel et méthodes \\ Données bioclimatiques du site}

La région physiographique de la vallée du Niari hébergeant le district de Madingou, représente $8 \%$ du territoire couverte d'une végétation savanicole (Vennetier, 1977). Située dans la partie orientale de la vallée du Niari, cheflieu de la Préfecture de la Bouenza, la sous-préfecture de Madingou est bordée par des collines calcaires et plateaux dont l'altitude varie de 200 à $700 \mathrm{~m}$ (Nzila, 1992). Les altitudes sont autour de 100 à $400 \mathrm{~m}$. Le bassin versant du fleuve Niari, situé entre 100 et $200 \mathrm{~m}$ d'altitude, arrose la sous-préfecture de Madingou via le Niari avec son affluent la Kenké. Sa situation géographique fait de lui un carrefour désenclavant les différentes localités de la Bouenza (Figure 1).

La région d'étude, située entre 4 et $5^{\circ}$ de latitude Sud, est soumise à un climat équatorial de transition dit "Bas-congolais" ou encore "Soudanoguinéen" (Aubréville, 1949 ; Samba-Kimbata, 1978). Ce climat est marqué par l'alternance de deux saisons (Figure 2). La saison de pluies, chaude et humide s'étale d'octobre à mai avec un net ralentissement de janvier à février. Deux pics de pluies sont relevés en (i) novembre et, (ii) aux mois de mars et avril. La moyenne de précipitation annuelle varie de 1000 à $1200 \mathrm{~mm}$. La saison sèche et fraîche court de juin à septembre (Vennetier, 1977).

Les températures moyennes annuelles sont de l'ordre de $25^{\circ} \mathrm{C}$. Les variations diurnes, saisonnières ou annuelles, faibles. L'hygrométrie relative moyenne est de l'ordre de $80 \%$ (Figure 3). L'humidité relative ne diminue que faiblement en saison sèche, alors que la température est minimale durant cette période. 


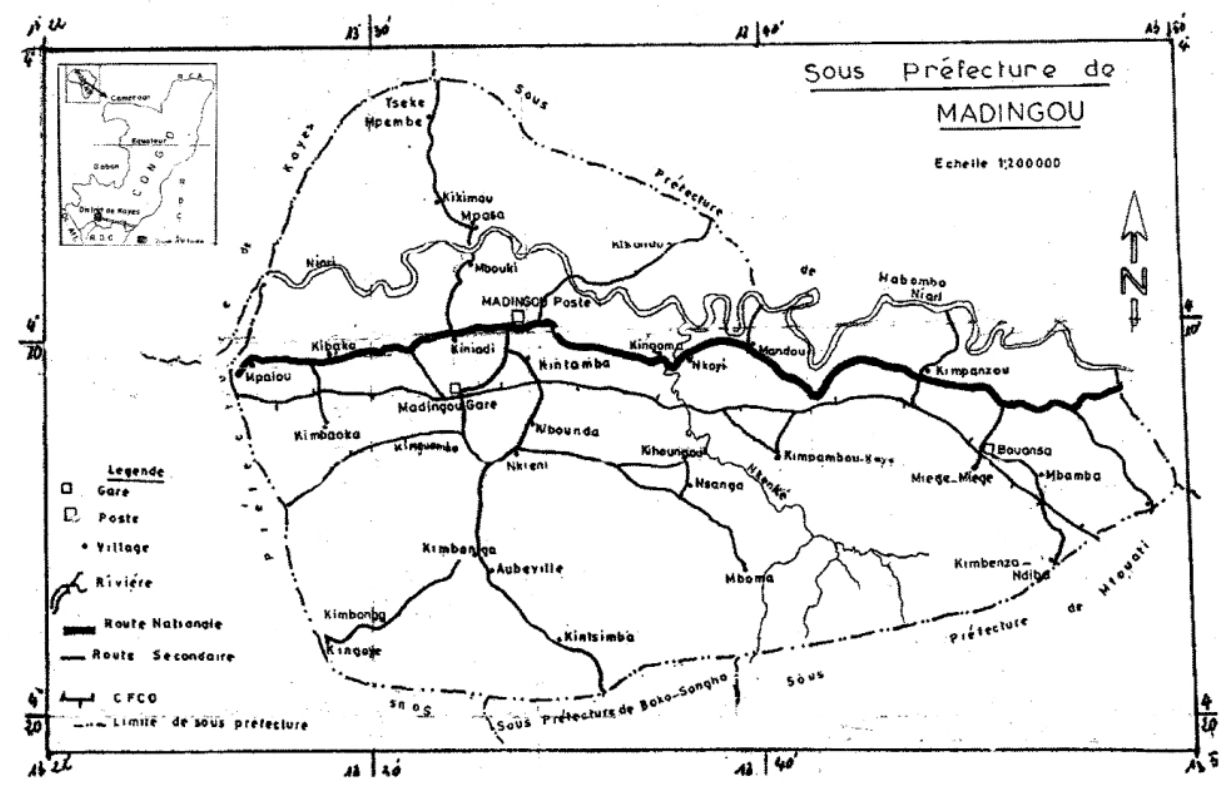

Figure 1. Localisation et situation géographique du District de Madingou

\section{Sol et végétation}

La géologie se base sur une formation continentale essentiellement constituée par la série Schisto-calcaire (Martin, 1962 ; Descoings, 1975 ; Nzila, 1992, 1996 ; Vennetier, 1977; Trochain, 1980). Les sols sont en général ferralitiques désaturés et pauvres en bases échangeables (Martin, 1962; Trochain, 1980 ; Nzila, 1992). La végétation de la vallée du Niari est une savane à Hyparrhenia sp. Elle est composée parfois d'un étage herbacé (Hyparrhenia sp., Andropogon sp., Panicum sp.) de 1,50 à $2 \mathrm{~m}$ et d'un étage arbustif de 2 à 5 mètres où l'on distingue Annona senegalensis, Bridelia ferruginea, et surtout Hymenocardia acida sur les sols schisto-calcaires. Les formations végétales subissent des dégradations significatives suites aux actions anthropiques: les feux de brousse annuels qui permettent le renouvellement de la végétation; et les activités culturales qui représentent un danger, surtout monoculturales. Elles n'affectent pas seulement la flore des terres cultivables mais dénature aussi le sol. Ces différents facteurs conduisent à une couverture imparfaite du sol l'exposant ainsi aux aléas des agents climatiques, notamment les précipitations et les vents.

\section{Population et activités économiques}

La population de Madingou en majorité paysanne, est de 62800 habitants pour une superficie de $590 \mathrm{~km}^{2}$, soit une densité de $106,4 \mathrm{hab} . / \mathrm{km}^{2}$ (CNSEE, 2010). Nonobstant les grandes villes (Brazzaville, Pointe-Noire, Dolisie et Nkayi), il reste le district le plus peuplé du Congo, avec une densité supérieure à la régionale $\left(25,2 \mathrm{hab} . / \mathrm{km}^{2}\right)$ et la nationale $\left(10,8 \mathrm{hab} . / \mathrm{km}^{2}\right)$. Le 
taux d'accroissement entre 1984 et 2007 est de 2,2\% pour la région de la Bouenza qui héberge le district de Madingou (CNSEE, 2010). Le district de Madingou, abrite deux communes urbaines qui sont : Madingou (25713 hab.) et Bouansa (19064 hab.). Notre étude a eu lieu en partie dans la commune urbaine et le district de Madingou.

La production agricole, essentiellement de subsistance, est portée sur les cultures vivrières (manioc, haricot, arachide, maïs, agrumes, légumes, petits pois, ignames, etc.). On y trouve parsemés des vergers de case formés des fruitiers. Outre l'agriculture, l'élevage paysan est l'une des activités économiques des populations. Pratiqué à proximité des habitations, sa base est : les petits ruminants (moutons et chèvres), les bovins, les porcs, la volaille, etc. En dépit d'un secteur primaire soutenu par la paysannerie, le secteur secondaire naguère florissant a fait faillite depuis plusieurs décennies (Mamboueni, 2017).

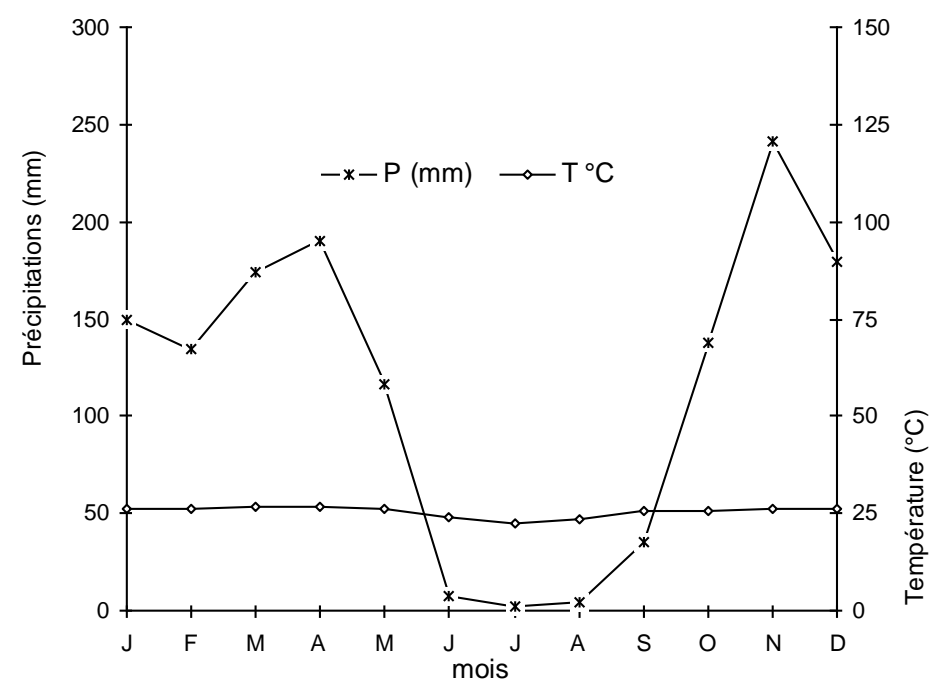

Figure 2. Diagramme ombrothermique de la zone d'étude

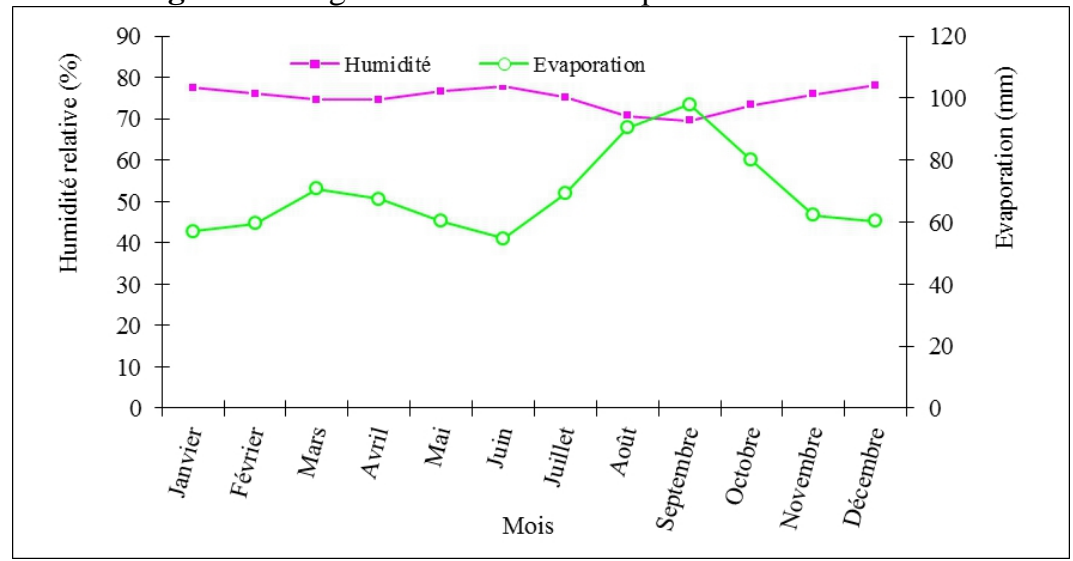

Figure 3 : humidité relative et évaporation au cours de l'année 


\section{Matériel}

Cette étude ethnobotanique se déroule en pleine saison sèche et couvre les mois de septembre à octobre 2015. A cette période où la majorité des espèces sont au repos physiologique, l'enquête ethnobotanique se focalise, sur la connaissance que les différents membres du groupe ethnique Kaamba possèdent sur les vertus de la flore avoisinante.

\section{Données sur les plantes médicinales}

Le matériel se compose des plantes à vocation médicinale, utilisées par le groupe ethnique Kaamba. L'identification des espèces est rendue possible par l'utilisation de plusieurs ouvrages qui font références à la flore africaine plus précisément ceux traitant des vertus phytothérapeutiques (Berhaut, 1967; Sofowara, 1996). Notons que les plantes les plus communes ont été identifiées in situ, alors que celles ayant présenté des difficultés, un recours est fait au Laboratoire de botanique de l'Ecole normale supérieure et le cas échéant à l'herbier national (IEC). Excepté quelques ajustements et traductions, les noms de différentes maladies et symptômes sont pris comme articulé par les informateurs. Les définitions et les classifications des problèmes de santé retenues obéissent aux normes de l'OMS (Adjanohoun et al., 1994). La classification des plantes suivie tient de l'APG III (Angiosperm phylogeny Group, 2009) et, la nomenclature taxinomique adoptée est de Lebrun et Stork (1991-1997).

Pour chaque spécimen indiqué, les ou la partie(s) utilisée(s) est notée(s), ainsi que les ou la maladie(s) et/ou les symptômes traités. Outre ces aspects, des informations sont recueillies sur le mode de préparation des médicaments, la posologie et le mode d'administration, d'une part, et d'autre part les éléments phytoécologiques et de gestion durable de la bioressource ont été relevés.

\section{Populations enquêtées}

A propos du groupe d'informateurs, suite à l'absence des guérisseurs et tradithérapeutes professionnelles, nous nous sommes penchés sur les personnes qui ont recours à ces pratiques au quotidien : c'est la pharmacopée populaire. Il est basé sur la mixité de genres et la valeur empirique qui sommeille en chacun d'eux. Son socle se compose principalement d'adultes et des personnes âgées ayant en charge des jeunes enfants. Le nombre d'informateurs n'est pas fonction de l'importance de la localité en termes de résident, mais plutôt en nombre d'individus possesseurs des connaissances ancestrales sur les plantes à vertus médicinales.

Notre échantillon d'informateurs regroupe 32 personnes dont 22 femmes possesseurs des connaissances sur les vertus des plantes. Il s'agit des 
personnes qui ont librement consenties et sans engagements de mettre à notre disposition leurs sommes de connaissances sur les savoirs endogènes.

\section{Méthodes}

La méthodologie est basée sur trois étapes qui sont complémentaires: (i) La revue bibliographique ; (ii) La collecte des données sur le terrain ; (iii) l'enquête ethnobotanique participative.

La revue bibliographique a permis de faire le point sur les études ethnobotaniques, connues dans le monde et particulièrement celles du territoire congolais. On note, que ces études présentent un caractère globalisant, qui ne fait pas ressortir les spécificités associées aux différents groupes ethnico-linguistiques.

La collecte d'information sur le terrain se subdiviser en deux parties : (i) les enquêtes qui s'appuient sur les entretiens personnalisés et les focus group impliquant les deux genres. Ces entretiens sont semi directifs et se basent sur un guide d'entretien à questions ouvertes, ce qui évite d'influencer la réponse des informateurs (Martin, 1995).

La démarche participative en ethnobotanique consiste à identifier les ressources et de connaitre les propriétés liées à ces plantes. Elle est ponctuée par les opérations de collecte des spécimens dans les vergers privés et dans la savane. Cette procédure permet de sérier et d'authentifier l'information reçue et si possible de la matérialiser en relevant les signes tangibles d'exploitation. Enfin, la démarche participative en ethnobotanique a consisté à suivre la collecte des spécimens, les modes de préparation des potions et d'administration, lorsque ce fut possible.

\section{Résultats}

\section{Données floristiques}

L'analyse de la diversité floristique révèle 80 espèces pour 72 genres (tableaux 1 et 2). Elles se répartissent dans 33 familles, dont les Fabaceae $(16,25 \%)$ sont plus représentatives. Les familles les moins représentées, avec une espèce, forment la majorité $(51,5 \%)$ et leur contribution respective est de $1,25 \%$. Cette diversité floristique couvre les aspects liés à la phytopharmacopée traditionnelle. Une analyse de la cohorte phytothérapeutique inventoriée montre que $26,5 \%$ sont allochtones et sont majoritairement cultivées. Quant aux 74,5\% restant, on relève des nitrophytes, des rudérales et des espèces des jardins de case et/ou vergers, et de la savane environnante.

L'usage des taxons allochtones et à large distribution est une preuve irréfutable d'acquisition et/ou appropriation de connaissances nouvelles, découlant des contacts avec d'autres sociétés. Les données 
phytogéographiques croisées aux usages médicinaux révèlent que ces plantes sont exploitées aux mêmes fins.

Tableau 1 : Récapitulatif taxinomique des taxons recensés.

\begin{tabular}{|c|c|c|c|c|c|c|}
\hline \multirow{2}{*}{ Groupes Angiospermes } & \multicolumn{3}{|c|}{ Niveau taxinomiques hiérarchiques } \\
\cline { 2 - 7 } & \multicolumn{2}{|c|}{ Familles } & \multicolumn{2}{c|}{ Genres } & \multicolumn{3}{c|}{ Espèces } \\
\cline { 2 - 7 } & Nombre & $\%$ & Nombre & $\%$ & Nombre & 61 \\
\hline Dicotylédones & 21 & 63,6 & 55 & 78,4 & 19 & 24 \\
\hline Monocotylédones & 12 & 36,4 & 17 & 23,6 & 80 & 100 \\
\hline Total & 33 & 100 & 72 & 100 & 80 & 76 \\
\hline
\end{tabular}

Tableau 2 : Synopsis des plantes utiles et des types d'usages recensés dans le terroir de Madingou associé au nombre de citation.

\begin{tabular}{|c|c|c|c|c|c|c|c|c|}
\hline $\begin{array}{c}\mathrm{N} \\
\circ\end{array}$ & Taxon & Famille & $\begin{array}{c}\text { Nom } \\
\text { vernaculaire }\end{array}$ & $\begin{array}{l}\text { Partie } \\
\text { utilisée }\end{array}$ & $\begin{array}{c}\text { Mode de } \\
\text { préparatio } \\
n\end{array}$ & $\begin{array}{c}\text { Mode } \\
\text { d'administratio } \\
n\end{array}$ & $\begin{array}{l}\text { Maladie ou } \\
\text { symptôme }\end{array}$ & $\begin{array}{l}\text { Nombre de } \\
\text { citation }\end{array}$ \\
\hline 1. & Mimosa pigra $\mathrm{L}$. & Fabaceae & Kikengui & Feuille & $\begin{array}{c}\text { Trituratio } \\
n\end{array}$ & $\begin{array}{c}\text { Voie } \\
\text { cutanée/frictio } \\
n ?\end{array}$ & Rougeole & $5(15,6 \%)$ \\
\hline 2. & $\begin{array}{l}\text { Kalonchoe crenata } \\
\text { (Anders.) Harv. }\end{array}$ & Crassulaceae & Yuka-yuka & Feuille & $\begin{array}{c}\text { Trituratio } \\
\mathrm{n}\end{array}$ & Voie rectale & Hémorroïdes & $1(3,1 \%)$ \\
\hline 3. & $\begin{array}{c}\text { Ageratum conyzoides } \\
\text { L. }\end{array}$ & Asteraceae & $\begin{array}{c}\text { lukaya lua } \\
\text { koki }\end{array}$ & Feuilles & $\begin{array}{c}\text { Trituratio } \\
\mathrm{n}\end{array}$ & Voie orale & Bronchite & $18(56,2 \%)$ \\
\hline 4. & Solanum melongena $\mathrm{L}$. & Solanaceae & $\begin{array}{c}\text { Binsoukoud } \\
\text { ou }\end{array}$ & Feuille & $\begin{array}{c}\text { Trituratio } \\
\mathrm{n}\end{array}$ & Voie orale & Bronchite & $18(56,2 \%)$ \\
\hline 5. & Boerhaavia diffusa L. & $\begin{array}{c}\text { Nyctaginacea } \\
\mathrm{e}\end{array}$ & Nsolokoto & $\begin{array}{c}\text { Racine } \\
\text { tubérisée }\end{array}$ & Pilat & voie orale & Bronchite & $18(56,2 \%)$ \\
\hline \multirow{2}{*}{6.} & \multirow{2}{*}{$\begin{array}{l}\text { Senna occidentalis (L.) } \\
\text { Link. }\end{array}$} & \multirow{2}{*}{ Fabaceae } & \multirow{2}{*}{ Kinkadimina } & Feuilles & \multirow{2}{*}{ Décoction } & \multirow{2}{*}{ voie orale } & Paludisme & \multirow{2}{*}{$5(15,6 \%)$} \\
\hline & & & & Racine & & & Fièvre jaune & \\
\hline
\end{tabular}




\begin{tabular}{|c|c|c|c|c|c|c|c|c|}
\hline 7. & Vitex madiensis Oliv. & $\begin{array}{c}\text { Verbenaceae / } \\
\text { Lamiaceae }\end{array}$ & Mfilou & $\begin{array}{c}\text { Feuilles } \\
\text { sèches }\end{array}$ & Décoction & Voie orale & $\begin{array}{l}\text { Hypotension } \\
\text { artérielle }\end{array}$ & $13(40,6 \%)$ \\
\hline 8. & Indet. & Indet. & $\begin{array}{l}\text { Singa } \\
\text { musitu }\end{array}$ & Racine & $\begin{array}{l}\text { Manger } \\
\text { les racines } \\
\text { crues }\end{array}$ & Voie orale & $\begin{array}{l}\text { Asthénie } \\
\text { sexuelle }\end{array}$ & $4(12,5 \%)$ \\
\hline 9. & $\begin{array}{l}\text { Sarcocephalus } \\
\text { latifolius (Smith) }\end{array}$ & Asteraceae & Mutumbi & Racine & $\begin{array}{c}\text { Macératio } \\
\mathrm{n} \text { avec } \\
\text { pour } \\
\text { solvant } \\
\text { l'eau }\end{array}$ & Voie orale & Paludisme & $14(43,7 \%)$ \\
\hline 10. & $\begin{array}{l}\text { Vernonia amygdalina } \\
\text { Del. }\end{array}$ & Asteraceae & $\begin{array}{l}\text { Munduri- } \\
\text { nduri }\end{array}$ & $\begin{array}{l}\text { Feuille, } \\
\text { Racine }\end{array}$ & Décoction & Voie orale & Typhoïde & $9(28,1 \%)$ \\
\hline \multirow[t]{2}{*}{11.} & \multirow[t]{2}{*}{ Moringa oleifera Lam. } & \multirow[t]{2}{*}{ Moringaceae } & \multirow[t]{2}{*}{ Murenga } & $\begin{array}{l}\text { Feuille, } \\
\text { Racine, } \\
\text { Ecorce, }\end{array}$ & décoction & Voie orale & \multirow{2}{*}{$\begin{array}{l}\text { Typhoïde } \\
\text { Paludisme } \\
\text { Mal de ventre } \\
\text { rhumatisme }\end{array}$} & \multirow[t]{2}{*}{$8(25 \%)$} \\
\hline & & & & Graines & $\begin{array}{l}\text { Consomm } \\
\text { ées crues }\end{array}$ & Voie orale & & \\
\hline 12. & Arachys hypogea $\mathrm{L}$. & Fabaceae & Nguba & Graines & $\begin{array}{l}\text { Consomm } \\
\text { ées crues }\end{array}$ & Voie orale & $\begin{array}{c}\text { Typhoïde } \\
\text { Paludisme } \\
\text { Mal de ventre } \\
\end{array}$ & $8(25 \%)$ \\
\hline 13. & Adansonia digitata $\mathrm{L}$. & Acanthaceae & Nkondo & Racine & Décoction & Voie orale & Dysenterie & $11(34,4 \%)$ \\
\hline 14. & $\begin{array}{c}\text { Momordica charantia } \\
\text { L. }\end{array}$ & Cucurbitaceae & $\begin{array}{l}\text { Dimbussi- } \\
\text { mbussi }\end{array}$ & Feuille & $\begin{array}{l}\text { Trituratio } \\
\text { n, } \\
\text { décoction }\end{array}$ & $\begin{array}{l}\text { Voie nasale et } \\
\text { la voie buccale }\end{array}$ & Asthme & $9(28,1 \%)$ \\
\hline 15. & $\begin{array}{c}\text { Desmodium } \\
\text { gangeticum } \mathrm{L} . D C .\end{array}$ & Fabaceae & Dintata & Feuille & $\begin{array}{l}\text { Piler, } \\
\text { Macéré }\end{array}$ & Voie orale & Diarrhée & $4(12,5 \%)$ \\
\hline 16. & $\begin{array}{l}\text { Aspilia kostchyi(Sch. } \\
\text { Bip.) Oliv. }\end{array}$ & Asteraceae & Mudjodi & Feuille & $\begin{array}{l}\text { Trituratio } \\
\text { n des } \\
\text { feuilles }\end{array}$ & Voie nasale & Céphale & $2(6,2 \%)$ \\
\hline
\end{tabular}




\begin{tabular}{|c|c|c|c|c|c|c|c|c|}
\hline 17. & Vernonia sp. & Asteraceae & $\begin{array}{l}\text { Lubangu- } \\
\text { bangu ya } \\
\text { kento }\end{array}$ & Feuille & $\begin{array}{c}\text { Mâcher } \\
\text { les feuilles } \\
\text { boire le } \\
\text { jus et } \\
\text { rejeté les } \\
\text { parties } \\
\text { restantes }\end{array}$ & Voie orale & $\begin{array}{c}\text { Douleurs } \\
\text { abdominales }\end{array}$ & $7(21,9 \%)$ \\
\hline 18. & $\begin{array}{c}\text { Saccharum } \\
\text { officinarum L. }\end{array}$ & Poaceae & $\begin{array}{c}\text { Canna à } \\
\text { sucre }\end{array}$ & Feuille & Décoction & Voie orale & $\begin{array}{c}\text { Tension } \\
\text { artérielle }\end{array}$ & $8(25 \%)$ \\
\hline 19. & $\begin{array}{c}\text { Desmodium } \\
\text { velutinum(Wilid.) DC. }\end{array}$ & Fabaceae & Lubata-bata & Feuille & $\begin{array}{l}\text { Contusion } \\
\text {, filtrat }\end{array}$ & Voie orale & Diarrhée & $9(28,1 \%)$ \\
\hline 20. & $\begin{array}{c}\text { Ocimum gratissimum } \\
\text { L. }\end{array}$ & Lamiaceae & $\begin{array}{l}\text { Mansusu- } \\
\text { susu }\end{array}$ & Feuille & $\underset{\mathrm{n}}{\text { Trituratio }}$ & $\begin{array}{l}\text { Voie nasale et } \\
\text { dans les yeux }\end{array}$ & Vertiges & $3(9,4 \%)$ \\
\hline 21. & Zea mays L. & Poaceae & Mansangu & $\begin{array}{l}\text { Stigmate } \\
\text { (style) }\end{array}$ & $\begin{array}{l}\text { Faire une } \\
\text { décoction } \\
\text { des styles } \\
\text { (stigmate) }\end{array}$ & Voie orale & Typhoïde & $9(28,1 \%)$ \\
\hline 22. & $\begin{array}{c}\text { Pennicetum } \\
\text { purpureum Schum. }\end{array}$ & Poaceae & Madiadia & Feuille & Décoction & Voie orale & Rhumatisme & $5(15,6 \%)$ \\
\hline 23. & Citrus limon L. & Rutaceae & Citron & Racine & Décoction & Voie orale & Paludisme & $18(56,2 \%)$ \\
\hline 24. & $\begin{array}{c}\text { Quisqualis hensii } \\
\text { (Enql. \& Diels) Exell }\end{array}$ & Combretaceae & $\begin{array}{l}\text { Lusumbi- } \\
\text { sumbi }\end{array}$ & Feuille & $\begin{array}{l}\text { Contusion } \\
\text { Macératio } \\
\text { n }\end{array}$ & Voie orale & Dysenterie & $4(12,5 \%)$ \\
\hline 25. & & & Muwissi & Feuille & contusion & Voie cutanée & Filariose & $2(6,5 \%)$ \\
\hline 26. & $\begin{array}{c}\text { Manihot esculenta } \\
\text { Crantz }\end{array}$ & $\begin{array}{c}\text { Euphorbiacea } \\
\mathrm{e}\end{array}$ & Ntomba & Feuille & Contusion & Voie cutanée & $\begin{array}{c}\text { Filariose ou } \\
\text { toute sorte de } \\
\text { démangeaiso } \\
\text { ns }\end{array}$ & $4(12,5 \%)$ \\
\hline 27. & $\begin{array}{c}\text { Bambusa vulgaris } \\
\text { Schrad. }\end{array}$ & Poaceae & Bambou & $\begin{array}{l}\text { Jeune } \\
\text { pousse }\end{array}$ & Décoction & Voie orale & $\begin{array}{l}\text { Tension } \\
\text { artérielle }\end{array}$ & $8(25 \%)$ \\
\hline
\end{tabular}




\begin{tabular}{|c|c|c|c|c|c|c|c|c|}
\hline 28. & Sida acuta Burm. F & Malvaceae & Ntumvumvu & Feuille & Contusion & Voie orale & $\begin{array}{c}\text { Maux } \\
\text { estomac }\end{array}$ & $2(6,5 \%)$ \\
\hline 29. & $\begin{array}{l}\text { Chromolaena odorata } \\
\text { (L) }\end{array}$ & Asteraceae & kandimina & Feuille & $\begin{array}{c}\text { Trituratio } \\
\mathrm{n}\end{array}$ & $\begin{array}{l}\text { Application } \\
\text { locale }\end{array}$ & Cicatrisation & $5(15,6 \%)$ \\
\hline 30. & $\begin{array}{c}\text { Lycopersicon } \\
\text { esculenta Mill. }\end{array}$ & Solanaceae & Tomate & Feuille & $\begin{array}{c}\text { Trituratio } \\
\mathrm{n}\end{array}$ & Voie nasale & Sinusites & $8(25 \%)$ \\
\hline 31. & $\begin{array}{c}\text { Alchornea cordifolia } \\
\text { Mull. Arg. }\end{array}$ & $\begin{array}{c}\text { Euphorbiacea } \\
\mathrm{e}\end{array}$ & Mubunzi & Feuille & Décoction & Voie orale & $\begin{array}{c}\text { Carie } \\
\text { dentaire }\end{array}$ & $2(6,5 \%)$ \\
\hline 32. & Terminalia catappa $\mathrm{L}$. & Combretaceae & Damier & Ecorce & Décoction & Voie orale & Dysenterie & $4(12,5 \%)$ \\
\hline 33. & $\begin{array}{l}\text { Acanthospermum } \\
\text { hispidium DC. }\end{array}$ & Asteraceae & $\begin{array}{c}\text { Madiata } \\
\text { mangombé }\end{array}$ & $\begin{array}{l}\text { Plante } \\
\text { entière }\end{array}$ & Contusion & Voie orale & $\begin{array}{c}\text { Lavage } \\
\text { abdominal }\end{array}$ & $1(3,1 \%)$ \\
\hline \multirow[b]{2}{*}{34.} & \multirow[b]{2}{*}{$\begin{array}{c}\text { Persea americanaa } \\
\text { Miller }\end{array}$} & \multirow[b]{2}{*}{ Lauraceae } & \multirow[b]{2}{*}{ Avocatier } & Feuille & Décoction & Voie orale & Toux & \multirow[b]{2}{*}{$13(40,6 \%)$} \\
\hline & & & & Noyau & Décoction & Voie orale & $\begin{array}{l}\text { Hypertension } \\
\text { artérielle }\end{array}$ & \\
\hline 35. & Annona muricata L. & Annonaceae & Corossol & Feuille & Décoction & Voie orale & Toux & $18(56,2 \%)$ \\
\hline 36. & $\begin{array}{c}\text { Eucalyptus citriodora } \\
\text { Hook. }\end{array}$ & Myrtaceae & Eucalyptus & Feuille & Décoction & Voie orale & Toux & $18(56,2 \%)$ \\
\hline \multirow{2}{*}{37.} & \multirow{2}{*}{$\begin{array}{l}\text { Dacryodes edulis } \\
\text { (G.Don) Lam. }\end{array}$} & \multirow{2}{*}{ Burseraceae } & \multirow{2}{*}{$\begin{array}{c}\text { Musafu } \\
\text { (Safoutier) }\end{array}$} & $\begin{array}{l}\text { Feuille } \\
\text { fraiche }\end{array}$ & Décoction & Voie orale & Toux & \multirow{2}{*}{$8(25 \%)$} \\
\hline & & & & $\begin{array}{l}\text { Feuille } \\
\text { sèche }\end{array}$ & $\begin{array}{l}\text { carbonisat } \\
\text { ion }\end{array}$ & $\begin{array}{l}\text { Application } \\
\text { locale }\end{array}$ & Teigne & \\
\hline 38. & Lantana camara L. & Verbenaceae & Lantana & Feuille & Décoction & Voie orale & Toux & $9(28,1 \%)$ \\
\hline 39. & Ficus sp. & Moraceae & Mussanda & Feuille & Décoction & Voie orale & Toux & $8(25 \%)$ \\
\hline 40. & Hybiscus sabdariffa L. & Malvaceae & Nkinkoula & Feuille & $\begin{array}{c}\text { Feuilles } \\
\text { ramollies, }\end{array}$ & $\begin{array}{l}\text { Application } \\
\text { locale }\end{array}$ & furonculose & $3(9,4 \%)$ \\
\hline 41. & $\begin{array}{c}\text { Vigna unguculata (L.) } \\
\text { Walpers }\end{array}$ & Fabaceae & Ndamba & Valves & $\begin{array}{l}\text { Carbonisa } \\
\text { tion }\end{array}$ & $\begin{array}{l}\text { Application } \\
\text { locale }\end{array}$ & furonculose & $5(15,6 \%)$ \\
\hline \multirow[t]{2}{*}{42.} & \multirow[t]{2}{*}{ Elaeis guineensis Jacq. } & \multirow[t]{2}{*}{ Arecaceae } & \multirow[t]{2}{*}{$\mathrm{Ba}$} & Fruit & $\begin{array}{c}\text { Trituratio } \\
\mathrm{n}\end{array}$ & Voie cutanée & $\begin{array}{l}\text { Douleurs } \\
\text { costales }\end{array}$ & \multirow[t]{2}{*}{$12(37,5 \%)$} \\
\hline & & & & Sève & Fermenté & Voie cutanée & Rougeole & \\
\hline
\end{tabular}




\begin{tabular}{|c|c|c|c|c|c|c|c|c|}
\hline & & & & Huile & Crue & Voie cutanée & Teigne & \\
\hline 43. & Musa x sapientum L. & Musaceae & Makonda & $\begin{array}{c}\text { Axe } \\
\text { infloresce } \\
\text { ntiel }\end{array}$ & $\begin{array}{c}\text { Mettre } \\
\text { dans l'eau } \\
\text { chaude } \\
\text { puis } \\
\text { massé }\end{array}$ & Voie cutanée & Fracture & $3(9,4 \%)$ \\
\hline 44. & Bidens pilosa $\mathrm{L}$. & Asteraceae & $\begin{array}{c}\text { nkongui nza } \\
\text { ngoulou }\end{array}$ & Feuilles & $\begin{array}{c}\text { Trituratio } \\
\mathrm{n}\end{array}$ & Voie cutanée & $\begin{array}{c}\text { Douleurs } \\
\text { costales }\end{array}$ & $6(18,7 \%)$ \\
\hline 45. & Citrus (L.) Osbeck. & Rutaceae & Oranger & Feuilles & Décoction & Voie orale & $\begin{array}{l}\text { Tête qui } \\
\text { chauffe }\end{array}$ & $1(3,1 \%)$ \\
\hline 46. & Gossypium hirsutum L. & Malvaceae & Cotonier & Feuille & $\begin{array}{c}\text { Trituratio } \\
\mathrm{n}\end{array}$ & Oreilles & Otite & $1(3,1 \%)$ \\
\hline 47. & $\begin{array}{c}\text { Cola nitida/ Cola } \\
\text { acuminata (P. Beauv.) } \\
\text { Scott \& Endl. }\end{array}$ & Malvaceae & Colatier & Noix & Manger & Voie orale & Hypotension & $10(31,2 \%)$ \\
\hline 48. & Spondias monbin L. & $\begin{array}{c}\text { Anacardiacea } \\
\mathrm{e}\end{array}$ & Mungengue & Feuille & Manger & Voie orale & Mastites & $12(37,5 \%)$ \\
\hline 49. & Senna siamea $(\mathrm{L})$. & Fabaceae & Acassia & Racines & Décoction & Voie orale & Typhoïde & $3(9,4 \%)$ \\
\hline 50 & Carica papaya $\mathrm{L}$. & Caricaceae & $\begin{array}{c}\text { Papayer } \\
\text { male }\end{array}$ & Racines & Décoction & Voie orale & $\begin{array}{l}\text { Asthénie } \\
\text { sexuelle }\end{array}$ & $6(18,7 \%)$ \\
\hline 51. & $\begin{array}{c}\text { Curculigo pilosa } \\
\text { (Schum. \& Thomn.) } \\
\text { Engl. }\end{array}$ & Hypoxidaceae & Ba dia nseke & $\begin{array}{c}\text { Racines } \\
\text { (tubercul } \\
\text { e) }\end{array}$ & Contusion & Voie cutanée & Panaris & $5(15,6 \%)$ \\
\hline \multirow{3}{*}{52.} & \multirow{3}{*}{$\begin{array}{c}\text { Millettia versicolor } \\
\text { Welw. Ex Back }\end{array}$} & \multirow{3}{*}{ Fabaceae } & \multirow{3}{*}{ Loubota } & Racines & Décoction & Voie orale & $\begin{array}{l}\text { Asthénie } \\
\text { sexuelle }\end{array}$ & \multirow{3}{*}{$12(37,5 \%)$} \\
\hline & & & & \multirow{2}{*}{ Feuilles } & Décoction & Voie orale & Constipation & \\
\hline & & & & & Contusion & Voie rectale & Rate & \\
\hline \multirow[t]{2}{*}{53.} & \multirow{2}{*}{$\begin{array}{l}\text { Gardenia ternifolia } \\
\text { Schum. Thonn }\end{array}$} & \multirow[t]{2}{*}{ Rubiaceae } & \multirow{2}{*}{$\begin{array}{l}\text { kilembe } \\
\text { nzau }\end{array}$} & Racines & Décoction & Voie orale & $\begin{array}{l}\text { Asthénie } \\
\text { sexuelle }\end{array}$ & \multirow[t]{2}{*}{$9(28,1 \%)$} \\
\hline & & & & Feuilles & Décoction & Voie orale & Gencivite & \\
\hline
\end{tabular}




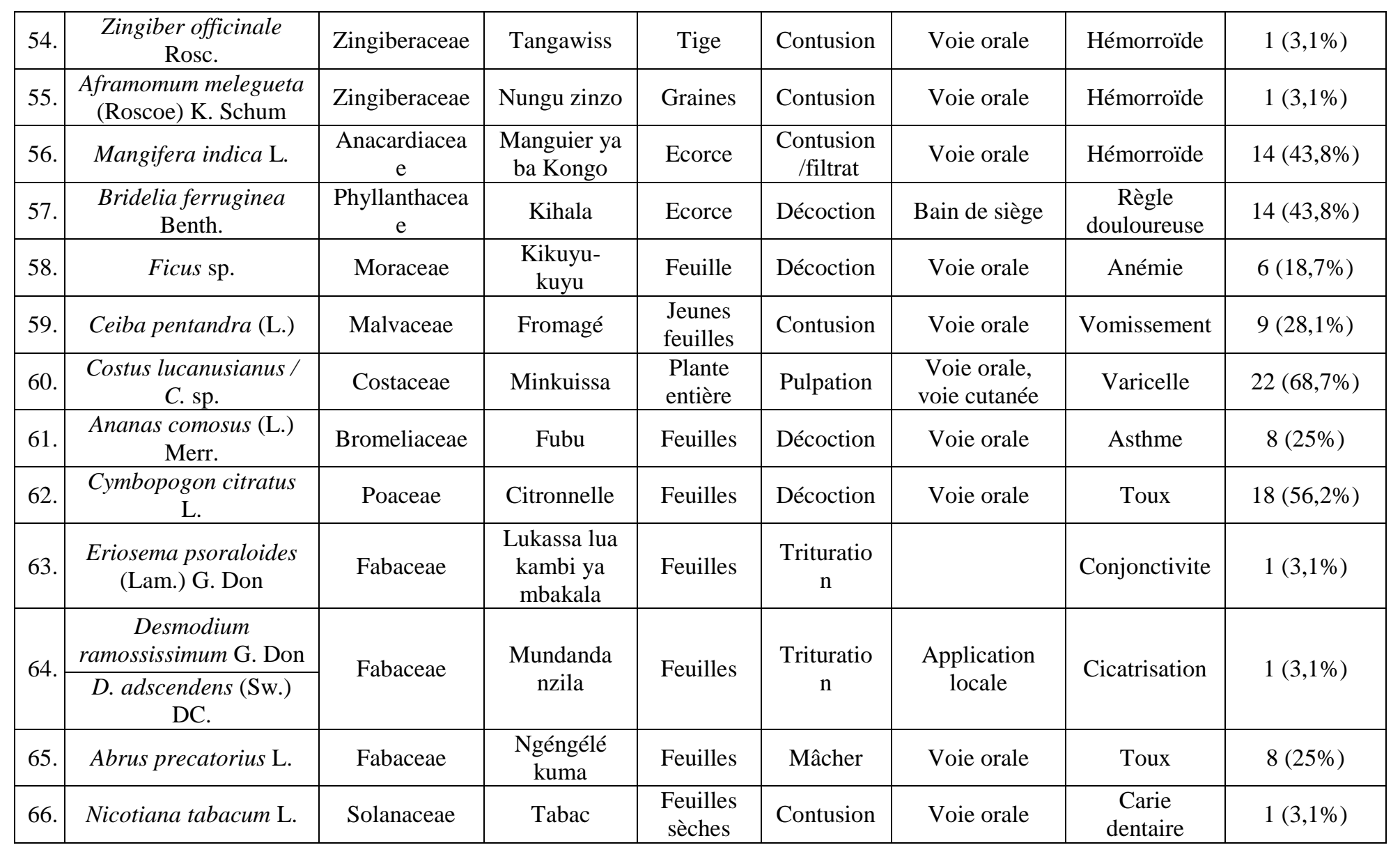




\begin{tabular}{|c|c|c|c|c|c|c|c|c|}
\hline 67. & $\begin{array}{c}\text { Leonotis nepetaefolia } \\
\text { Var. africana } \\
\text { J.K.Morton }\end{array}$ & $\begin{array}{l}\text { Verbenaceae / } \\
\text { Lamiaceae }\end{array}$ & $\begin{array}{l}\text { Malamu ma } \\
\text { songui }\end{array}$ & Feuilles & $\begin{array}{c}\text { Trituratio } \\
\mathrm{n}\end{array}$ & Voie nasale & Maux de tête & $7(21,9 \%)$ \\
\hline 68. & $\begin{array}{l}\text { Titonia diversifolia } \\
\text { (Hem.) A. Gray }\end{array}$ & Asteraceae & Nduri-nduri & Feuilles & $\begin{array}{l}\text { Contusion } \\
\text { / filtrat }\end{array}$ & $\begin{array}{l}\text { Voie cutanée, } \\
\text { Voie orale }\end{array}$ & ascaridiose & $6(18,7 \%)$ \\
\hline 69. & $\begin{array}{l}\text { Crossopterix febrifuga } \\
\text { Benth. }\end{array}$ & $\begin{array}{c}\text { Verbenaceae/ } \\
\text { Lamiaceae }\end{array}$ & Mukakati & Ecorce & Décoction & Voie orale & $\begin{array}{c}\text { Diarrhée } \\
\text { amibienne }\end{array}$ & $13(40,6 \%)$ \\
\hline 70. & $\begin{array}{c}\text { Brillantaisia patula } \mathrm{T} . \\
\text { Anders. }\end{array}$ & Acanthaceae & $\begin{array}{c}\text { Lemba- } \\
\text { lemba }\end{array}$ & Feuilles & $\begin{array}{c}\text { Trituratio } \\
\mathrm{n}\end{array}$ & Voie orale & Asthme & $8(25 \%)$ \\
\hline 71. & Alium sativum $\mathrm{L}$. & Alliaceae & Ail & & $\begin{array}{l}\text { Contusion } \\
\text { /filtrat }\end{array}$ & Voie cutanée & Varicelle & $22(68,7 \%)$ \\
\hline 72. & $\begin{array}{l}\text { Citrus paradisii } \\
\text { Macfad. }\end{array}$ & Rutaceae & $\begin{array}{c}\text { Pamplemous } \\
\text { se }\end{array}$ & Fruits & Succion & Voie orale & $\begin{array}{l}\text { Hypotension } \\
\text { artérielle }\end{array}$ & $8(25 \%)$ \\
\hline 73. & Morinda lucida Benth. & Rubiaceae & Mussiki & $\begin{array}{l}\text { Feuilles, } \\
\text { Racines }\end{array}$ & Décoction & Voie orale & Rougeole & $5(15,6 \%)$ \\
\hline 74. & $\begin{array}{c}\text { Pentaclethra } \\
\text { macrophylla Benth. }\end{array}$ & Fabaceae & Mpanzu & Valves & Décoction & Bain de siège & & $3(9,4 \%)$ \\
\hline 75. & $\begin{array}{l}\text { Millicia excelsa } \\
\text { (welw.) C. Berg }\end{array}$ & Moraceae & Mbuyu & Feuilles & Décoction & Voie orale & Mastite & $12(37,5 \%)$ \\
\hline 76. & Abrus sp. & & Nguenga & Feuilles & Contusion & Voie orale & Toux & $1(3,1 \%)$ \\
\hline 77. & $\begin{array}{l}\text { Newbouldia laevis } \\
\text { (P.Beauv.) Seem. }\end{array}$ & Bignoniaceae & $\begin{array}{l}\text { Mpéssé- } \\
\text { mpéssé }\end{array}$ & Ecorce & $\begin{array}{c}\text { Macératio } \\
n\end{array}$ & Voie orale & Estomac & $1(3,1 \%)$ \\
\hline 78. & $\begin{array}{l}\text { Annona senegalensis } \\
\text { var. arenaria (Thonn.) } \\
\text { Sillans }\end{array}$ & Annonaceae & Lolo nséké & $\begin{array}{l}\text { Feuilles, } \\
\text { écorce }\end{array}$ & $\begin{array}{l}\text { Décoction } \\
\text {, pilat }\end{array}$ & $\begin{array}{l}\text { Voie orale, } \\
\text { application } \\
\text { locale }\end{array}$ & $\begin{array}{l}\text { Anémie, } \\
\text { hémorragie }\end{array}$ & $7(21,9 \%)$ \\
\hline 79 & Psidium guajva $\mathrm{L}$. & Myrtaceae & Goyavier & Feuilles & Décoction & Voie orale & $\begin{array}{l}\text { Diarrhée } \\
\text { amibienne }\end{array}$ & $13(40,6 \%)$ \\
\hline 80 & Crinum cf. zeylanicum & $\begin{array}{c}\text { Amaryllidace } \\
\text { ae }\end{array}$ & Halaba & Bulbe & Décoction & Voie orale & Rhumatisme & $1(3,1 \%)$ \\
\hline
\end{tabular}




\section{Données d'exploitation de la phytodiversité}

L'enquête ethnobotanique révèle 80 espèces utilisées pour soulager les divers symptômes et maladies (tableau 2). Des 9 types d'organes utiles, les feuilles $(65 \%)$ sont les plus utilisées, devant les racines et les rhizomes (figure 4A). Les organes les moins utilisés ont une contribution de $1,25 \%$ et correspondent à $15,15 \%$ des familles.

Suivant les vertus phytothérapeutiques, $8,75 \%$ des plantes fournissent plus d'un organe (figure 4B). Les plantes les plus en vue sont celles qui fournissent les (i) feuilles et, (ii) les feuilles et les racines. Celles donnant les racines et rhizomes n'arrivent qu'en $3^{\mathrm{e}}$ position. Quel que soit le niveau d'usage, le taux de citation montre que les vertus phytothérapeutiques associées auxdites plantes sont bien connues et exploitées par la population, notamment féminine. Plus de $2 / 3$ des plantes médicinales sont citées par les femmes d'âge mature et en charge des enfants.

Les plantes ayant un nombre de citations au moins égal à $50 \%$ représentent 11,25\% de l'inventaire et ce sont: Ageratum conyzoides, Boerhaavia diffusa, Solanum melongena, Citrus limon, Anonna muricata, Eucalyptus citriodora, Cymbopogon citratus, Costus lucanusianus, Allium sativum.

\section{Méthodes de préparation et d'administration des potions}

Le mode de préparation des potions médicamenteuses le plus usité est la décoction $(47,5 \%)$. Elle est suivie presque de moitié par la trituration et la contusion (Figure 5A). Les éléments médicinaux issus de ces plantes peuvent être associés, dans les recettes, aux substances minérales, animales voire des adjuvants ou des excipients.

En rapport avec le mode d'administration, la voie orale est la plus répandue, eu égard au fait que la grande majorité des potions se présente sous la forme de «tisane ». Toutefois, une représentativité adéquate est relevée pour les potions médicamenteuses associées à la voie cutanée, instillations nasales et otique (Figure 5B).

\section{Maladies et/ou symptômes traités}

L'enquête met en relief 43 maladies et symptômes traités par les 80 taxons recensés (Tableau 2, Figure 6). L'analyse du nombre de citations montre une nette dominance des indications antitussives, avec 9 (soit 11,25\%) plantes dont la fréquence de citation est de 8 à $18 \%$, excepté Abrus sp. $(3,1 \%)$. Retenons la prise en compte des maladies comme l'hypertension artérielle (6 plantes, soit 7,5\%), le paludisme et la fièvre typhoïde avec 5 plantes chacune, soit $6,25 \%$. Quant aux autres, elles, ont un faible nombre d'espèces. Toutefois, relevons que le faible nombre d'espèces inhérent à une maladie n'implique pas nécessairement une faible fréquence de citations et donc d'usage. Le 
regroupement par sphère d'organes et/ou de maladies met en évidence les maladies infectieuses et parasitaires. Elles sont suivies par les symptômes et signes mal définis, et les maladies de l'appareil digestif (Figure 6).

Le regroupement en fonction des sphères de maladies et des organes décline 11 grandes affiliations à cette zone d'étude. Les maladies infectieuses et parasitaires de par leur contribution $(23,25 \%)$ sont les plus courantes (Figure 7). Les sphères de maladies les moins représentées sont à 2,33\%.

Figure 4. Contribution des organes usités et typification par espèce

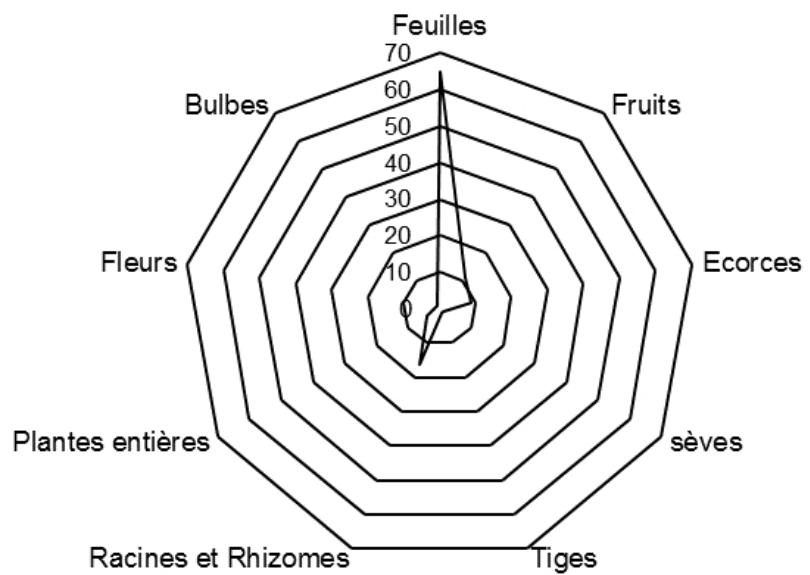

Proportion d'intervention des organes usités

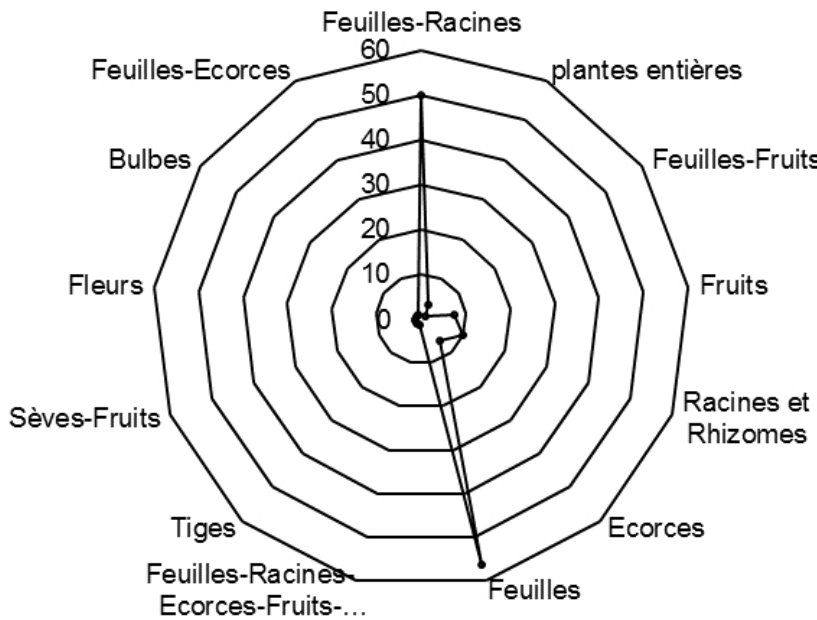

Contribution des plantes par types d'organes exploités 
Figure 5. Mode de préparation et voies d'administration des potions

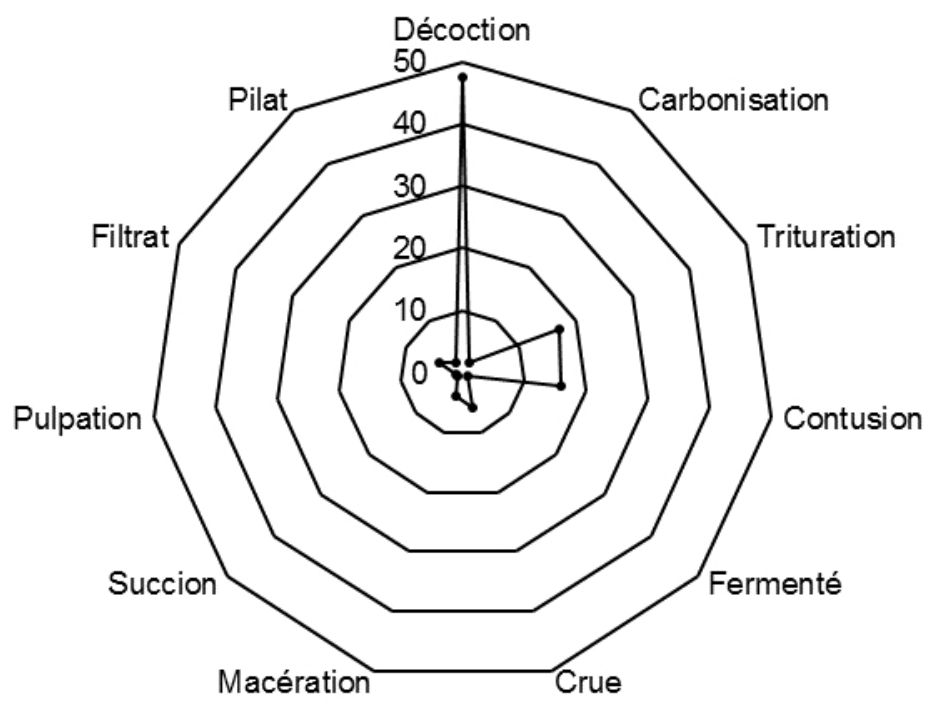

Mode de préparation des phytomédicaments

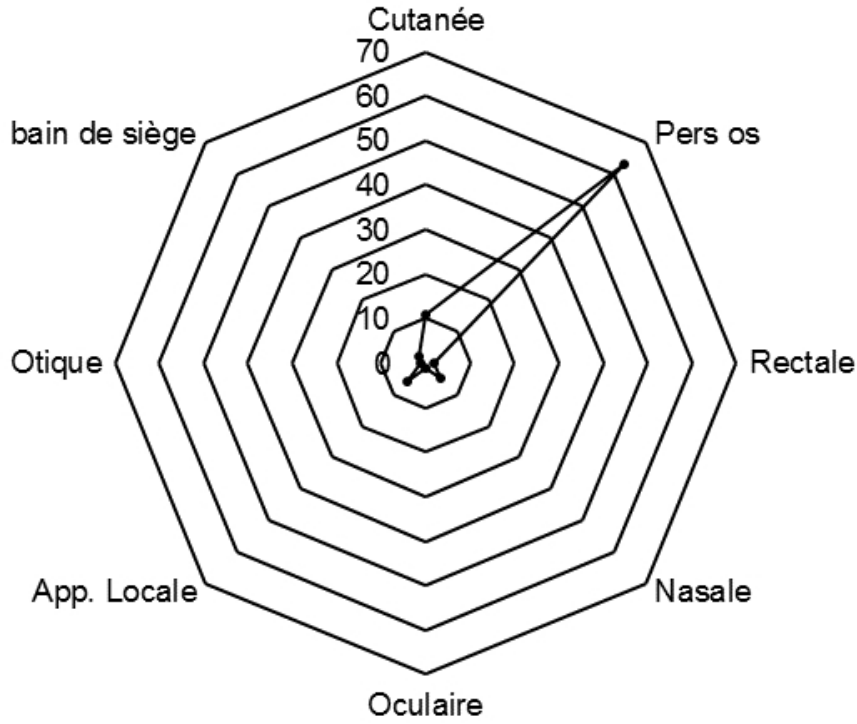

Voies d'administration des phytomédicaments 
Figure 6. Taux indications thérapeutiques suivant les groupes de maladies et des organes.

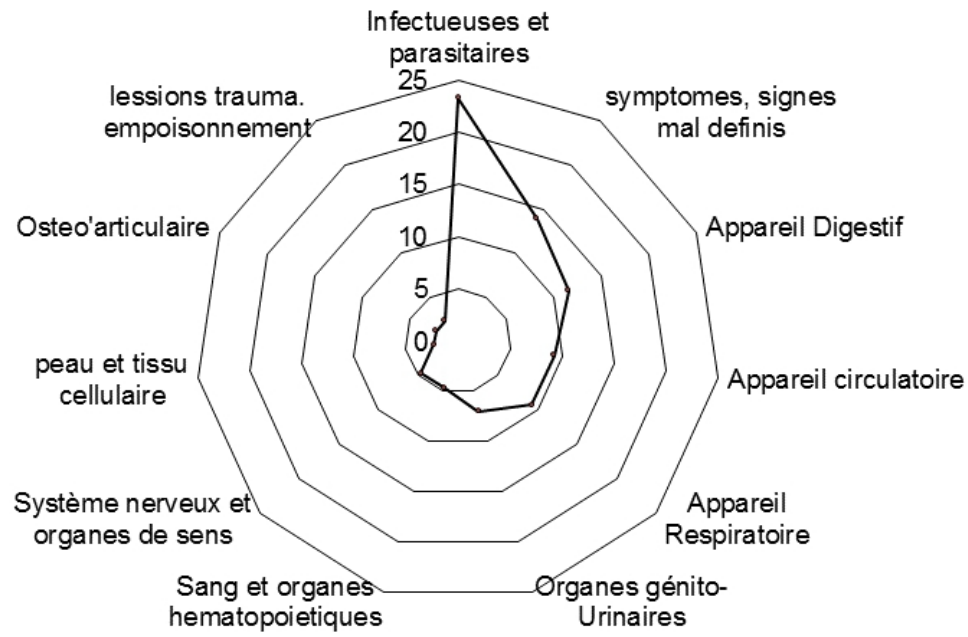

Figure 7 : Rapport des citations plantes et maladies par informateurs.

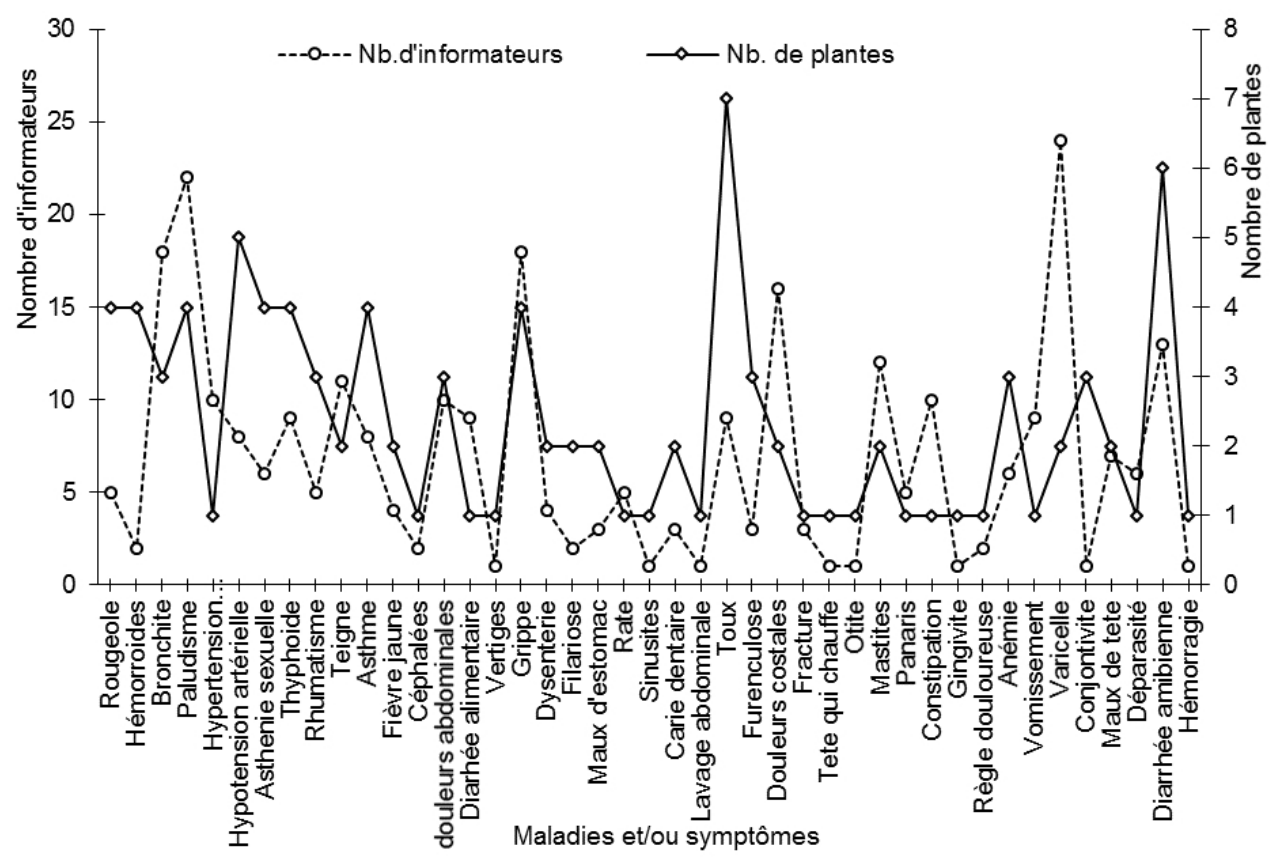

\section{Discussion}

\section{Diversité floristique}

L'analyse des résultats montre que, cette pharmacopée traditionnelle est liée essentiellement aux plantes autochtones. L'intérêt affecté à ces plantes au quotidien, prouvent qu'il existe un lien intime entre les populations et elles (Tailfer, 1989 ; Grenand \& Prevost, 1994 ; Emperaire \& Lescure, 1994 ; 
Kimpouni \& Nguembo, 2007). Les savoirs endogènes sur les plantes allochtones résultent des circonstances telles que : le brassage des groupes ethnico-linguistiques, qui permet le transfert de connaissances. En outre, elles peuvent découler de l'exploitation des données livresques sur les savoirs endogènes des peuples des régions d'origine (Kimpouni \& Motom, 2006 ; Mpassi, 2007 ; Nkoukou Loufoukou, 2012). Au niveau de notre site d'étude, quoique le nombre de plantes recensées soit faible et un nombre d'informateurs limité, les connaissances empiriques restent vivaces, surtout au sein du genre féminin (Wezel, 2002; Sanogo, 2006 ; Dupius, 2011). Cette spécificité serait peut être associée au caractère matrilinéaire de cette société.

L'ensemble des plantes, à la base de cette étude, a fait l'objet de citations phytothérapeutiques au Congo et ailleurs, notamment des groupes ethnico-linguistiques de leur aire géographique (Raponda-Walker \& Sillans, 1961 ; Bouquet, 1969 ; Adjanohoun et al., 1988 ; Hecketsweiler et al., 1991 ; Kimpouni, 1999; Kimpouni et al., 2012). Cette large reconnaissance et exploitation des vertus médicinales desdites plantes, est la preuve avérée de leur utilité dans les relations de l'homme et son environnement. Des espèces utilisées par la population Kaamba, 52 espèces (soit 65\%) sont strictement médicinales ; tandis que, les 28 (soit $35 \%$ ) autres, nonobstant leurs propriétés phytothérapeutiques, sont aussi citées comme alimentaires, via les mêmes organes (Kimpouni \& Motom, 2012). Cette donnée est la parfaite illustration d'une maîtrise desdits taxons par la population.

Une analyse des données ethnobotaniques révèle que certaines espèces (Ficus sp., Saccharum officinarum, Zea mays) sont rarement signalées comme des plantes médicinales, alors qu'elles le sont très souvent dans la pharmacopée traditionnelle africaine. Des espèces recensées dans la dition, Crinium cf. zeylanicum est celle qui n'a jamais été citée comme médicinale au Congo (Bouquet, 1969 ; Adjanahoun et al., 1988 ; Hecketsweiler et al., 1991). Cette première citation de Crinium $c f$. zeylanicum dans la pharmacopée traditionnelle congolaise est un apport spécifique à cette communauté.

\section{Diversité floristique et actions anthropiques}

Les actions anthropiques restent les causes des modifications de la diversité floristique. Dans la contrée, elles se déclinent comme suit: l'urbanisation anarchique ne répondant à aucun plan directeur d'urbanisme, l'édification des infrastructures physiques sans étude d'impact environnemental et social préalable. A cette cohorte d'actions anthropiques, le rôle de (i) l'agriculture itinérante sur brûlis, (ii) la pression démographique sur les terres agricoles, (iii) le caractère violent des feux de brousses récurrents et intervenant en fin de saison sèche, sont une donne non négligeable dans la vulnérabilité qui caractérise ces taxons, à travers la destruction des habitats. Les feux ont un effet sélectif sur la flore en favorisant le développement des 
pyrophytes (pyroclimax) au détriment de toutes les autres qui sont éradiquées (Bergonzini \& Lanly, 2000 ; Kimpouni et al., 2013, 2014). Ceci est plus vrai que plusieurs d'entre elles se retrouvent désormais dans les vergers et les jardins de cases.

La raréfaction voire l'extinction des espèces est l'une des raisons de la résiliation de certaines recettes de la pharmacopée traditionnelle. C'est le cas du Senna alata (Datrier), reconnu de par le monde et dans la dition comme antimycose (Makany, 1976 ; Sofowora, 1996 ), dont la population regrette les vertus. Ce phénomène abouti à l'étiolement des savoirs endogènes au sein de la société. Sous-tendus par l'empirisme, ces acquis sont des valeurs intrinsèques du socle social traditionnel (Kimpouni, 2001; Kimpouni \& Motom, 2011, 2012). Malgré les effets précités, les feux contribuent efficacement à l'érosion génétique de l'écosystème suite aux modifications des niches écologiques et en détruisant les éléments (spores, graines) de pérennisation des plantes (Bergonzini \& Lanly, 2000 ; Frontier et al., 2008 ).

\section{Analyse ethnophytogéographique}

Les données de la répartition géographique montrent que plusieurs espèces citées sont bien connues de par le monde comme médicinales. Les taxons visés sont Ageratum conyzoüdes, Boerhaavia diffusa, Vitex madiensis, Sarcocephalus latifolius, Citrus limon, Mangifera indica, Costus lucasianus, Cymbopogon citratus, Crossopterix ferbrifuga, Psidium guyava, Bridelia ferruginea (Berhaut, 1967 ; Bouquet, 1969 ; Adjanohoun et al., 1988 ; Lavergne \& Vera, 1989 ; Sofowra, 1996). Les variations d'usage observées seraient des spécificités associées à l'empirisme et aux communautés ethnicolinguistiques. C'est ainsi que : (i) Carica papaya (Caricaceae) traite l'asthénie sexuelle, selon les études menées au Cameroun et au Ghana (Saotoing et al., 2011), au Nigeria (Idowu et al., 2010), à la Réunion (Lavergne \& Vera, 1989), au Gabon (Betti et al., 2013a, 2013b). Des nouvelles utilisations ont été signalées dans les mêmes contrées, comme les difficultés d'accouchement, le traitement du paludisme ; (ii) Cymbopogon citratus (Poaceae) usité comme un antitussif, est signalé par Titanji et al. (2008) au Cameroun, Idowu et al. (2010) au Nigeria, Betti et al. (2013a, 2013b) au Gabon. Outre cette indication, on lui associe le pouvoir de soulager l'angine, l'asthme ; (iii) Vernonia amygdalina (Asteraceae) reconnue comme remède contre la fièvre typhoïde dans la dition l'est aussi au Cameroun (Hakizamungu \& Weri, 1988 ; Iwu, 1993 ; Idowu et al., 2010), au Gabon (Betti et al., 2013a, 2013b), tout en lui associant le traitement du paludisme; (iv) Zingiber officinale (Zingiberaceae) est citée contre les hémorroïdes chez les Kaamba et la Réunion (Lavergne \&Vera, 1989). Dans la même étude, elle serait à la base des traitements des fièvres, des rhumatismes, des douleurs musculaires, des blessures; (v) Lantana camara (Verbenaceae) très répandue et connue pour ses propriétés 
pharmaceutiques, contre la grippe, est aussi citée par Lavergne \& Vera (1989) à La Réunion. Outre la grippe on la retrouve dans le traitement de la toux ; (vi) Kanlanchoe crenata (Crassulaceae) citée pour traiter les hémorroïdes est reprise en Ouganda (Dalziel, 1956). Outre le traitement des hémorroïdes, elle est également citée pour la gonorrhée, faiblesse générale, convulsions, palpitation, asthme, antidote des poisons; (vii) Momordica charantia (Cucurbitaceae) reconnue pour l'asthme est signalée pour une même fin à Porto-Rico (Rivera, 1941), aux Philippines (Dalziel, 1956 ; Watt \& BreyerBandwijk, 1962). On ajoute dans son spectre d'action le traitement des troubles gastro-intestinaux, antidiabétique, antihelminthiques (Bouquet, 1969; Adjanohoun et al., 1988 ; Sofowora, 1996).

En tenant compte de la valeur pharmaceutique, certaines plantes ont fait l'objet de screening chimique, pour l'isolement des agents bioactifs utilisés dans la médecine moderne, afin de synthétiser des nouvelles molécules ; la fabrication des médicaments plus efficace et de toxicité moindre (Bouquet, 1972; Sofowara, 1996). Parmi elles, nous citons Cymbopogon citratus, Nicotiana tabacum, Bridelia ferruginea, Momordica charantia, Moringa oleifera (Olivier, 1959 ; Jain \& Sharma, 1967 ; Ampofo, 1977 ; Saluja et al., 1978 ; Eilert et al., 1980 ; Ramachandran et al., 1980 ; Adesina, 1982 ; Iwu, 1983 ; Sofowara, 1996). La plante qui compte de nombreuses citations dans la pharmacopée traditionnelle Kaamba et dont le screening chimique est suffisamment abouti est le Cymbopogon citratus.

\section{Analyse ethnobotanique}

Les données ethnobotaniques du Congo, font la part belle aux zones forestières, comme si les populations savanicoles n'accordent pas une attention particulière à la biodiversité environnante. Les acquis de cette étude établissent que les peuples de savanes usent parfaitement de la biodiversité à portée de la main. Toutes les espèces répertoriées au cours de cette recherche sont bien connues pour leurs propriétés pharmacognosiques (Bouquet, 1969 ; Makany, 1976; Adjanohoun et al., 1988 ; Profizi et al., 1993 ; Sofowara, 1996). L’observation de cette donnée étale une différence entre populations forestière et savanicole. Les écorces et les racines sont plus prisées en zone forestière, contre les feuilles en savane. Ces choix s'imposent naturellement à eux, eu égard aux types écosystèmes et de phytodiversité en présence.

Une analyse approfondie de l'inventaire floristique souligne que 15\% des espèces recensées, comptent parmi les plus utilisées en pharmacopée traditionnelle, par les communautés africaines (Sofowora, 1996). Il s'agit de : Kalanchoe crenata, Boerhaavia diffusa, Arachys hypogea, Momordica charantia, Ocimum gratissimum, Spondias monbin, Carica papaya, Zingiber officinale, Cymbopogon citratus, Nicotiana tabacum, Allium sativum, Morinda lucida. Une consultation de l'extrait de la banque des données 
NAPRALERT, sur la cinquantaine des plantes apparaissant 50 fois et plus, met en évidence trois taxons (Allium sativum, Persea americana, Solanum nigrum) recensés dans notre étude. Ces plantes correspondent à 17\% de l'inventaire et ont une fréquence de citation d'au moins $40 \%$ dans la dition. Les deux premières sont des plantes introduites et domestiquées. L'Allium sativum qui n'est pas une plante cultivée au Congo, n'est disponible que dans le commerce. Persea americana introduite du néotropis est une plante cultivée dans le pays. Le Solanum nigrum, est une plante spontanée et autochtone de la zone d'étude.

Les données des indications thérapeutiques sur les maladies ou symptômes traités dans la zone, déterminent une large dominance des maladies infectieuses et parasitaires, comme c'est généralement partout ailleurs au Congo (Kimpouni et al., 2012; Bokatola, 2013). Le taux de prévalence de cette sphère de maladies serait lié aux problèmes d'hygiène, d'assainissement du milieu et du manque d'eau potable.

Dans le traitement des maladies et symptômes, les feuilles, les écorces, les racines et les fruits sont les parties les plus recherchées. Le prélèvement desdits organes peut durement affecter les taxons exploités voire l'écosystème, si la fréquence s'élève et/ou que les quantités prélevées augmentes (Peters, 1997 ; Ticktin, 2004). La collecte des organes végétatif et génératif impacte la stabilité, la dynamique, la structure et la régénération naturelle des écosystèmes, surtout les formations ligneuses (Peters, 1997 ; Ticktin, 2004 ; Betti, 2013a, 2013b).

\section{Conclusion}

Cette étude met en relief des informations utiles à la science et aux générations futures, dépositaires des savoirs endogènes; socle socioculturel de chacune des communautés congolaises et d'ailleurs. Suite aux attraits mondains synonymes du bien-être social, la communauté Kaamba assiste stoïquement à la dégradation de son socle socioculturel. En effet, la pyramide des âges se renverse à propos des savoirs endogènes, car les jeunes garçons qui seraient les dépositaires des savoirs représentent la minorité des informateurs voire de la population. L'étude révèle que le centre de gravité des savoirs se déplace vers le genre féminin, corollaire de la pression qu'exerce la société urbaine sur les jeunes garçons principalement, à la recherche d'une vie meilleure.

Aussi, cette société matrilinéaire, est très stricte sur l'éducation de la femme, régente du foyer. La manifestation de ce fait est le grand nombre de recettes délivrées par des femmes ayant des enfants à charge. Ces recettes ciblent principalement les maladies ou symptômes inhérents aux enfants. Cette vision traduit le déséquilibre des genres, au sein du groupe d'informateurs, et explique la prépondérance des femmes âgées, sans une relève. 
L'analyse floristique a montré la richesse et la qualité de la flore de Madingou, juste un petit point comparé l'aire ethnico-linguistique Kaamba. Elle est aussi une référence pour la région de la Bouenza et la vallée du Niari dont la couverture végétale est dominée par la savane. Consécutivement à l'érosion des savoirs, la flore utile à la pharmacopée traditionnelle se retrouve de plus en plus sous la menace d'extinction, suite aux actions anthropiques sans communes mesures.

En dépit des aléas que connaît la société Kaamba de Madingou, la maîtrise des vertus de la diversité végétale environnante, en matière de pharmacopée traditionnelle, est une réalité pour les populations de savanes. Le recours à la domestication peut être interprété comme la preuve des limites des écosystèmes naturels, d'une part, et d'autre part, d'une intégration des savoirs extrinsèques au socle socioculturel.

\section{References:}

1. Adesina, S.K. (1982). Studies on some plants used as anticonvulsants in Amerindian and African Traditional medicine. Fitoterapia, 53, pp 147-162.

2. Adjanohoun, E.J., Ahyi, A.M.R., Ake Asi, L., Baniakina, J., Chibon, P., Cusset, G., Doulou, V., Enzanza, A., Eyme, J., Goudote, E., Keita, E., Mbemba, C., Mollet, J., Moutsamboté, J.-M., Mpati, J. \& Sita, P. (1988). Contribution aux études ethnobotaniques et floristiques en République Populaire du Congo: Médecine traditionnelle et pharmacopée. ACCT, Paris (France).

3. Adjanohoun, E.J., Cusset, G., Issa, LO, Keita, A., Lebras M., Lejoly, J. (1994). Banque de données de Médicine traditionnelle et Pharmacopée (PHARMEL): Notice pour la collecte et l'entrée des données, 2 éd. ACCT, Paris, (France).

4. Ampofo, O. (1997). Some clinical observations of the treatment of selected diseases by herbal preparations, Perspectives in medecinal plant research today. Obafemi Awolowo University, Ile-Ife, Nigeria, pp 35-45.

5. APG III. (2009). An update of the Angiosperm phylogeny group classification for the orders and families of flowering plants: APG III. Bot. J. Linn. Society, 161, pp 105-121.

6. Aubréville, A. (1949). Climats, forêts et désertification de l'Afrique tropicale. Société d'Éditions Géographiques, Maritimes et Coloniales, Paris (France).

7. Baker, J.T., Borris, R.P., Carte, B., Cordell, G.A., Soejarto, D.D., Cragg, G.M., Gupta, M.P., Iwu, M.W., Maduud, D.R. \& Tyler, V.E. (1995). Natural product drug discovery and development: new 
perspectives on international collaboration, J. Nat. Prod., 58, pp 13251357.

8. Bergonzini, J.-C. (2004). Changements climatiques, désertification, diversité biologique et forets. Silva, RIAT, Paris (France).

9. Bergonzini, J.-C. \& Lanly, J.-P. (2000). Les forêts tropicales. Paris: Karthala, CIRAD.

10. Berhaut, J. (1967). Flore du Sénégal, 2è Ed. Clairafrique, Dakar (Sénégal).

11. Betti, J.-L., Yongo, O.D., Obiang Mbomio, D., Midoko Iponga D. \& Ngoye A. (2013a). An ethnobotanical and floristical study of medicinal plants among the Baka pygmies in the periphery of the IpassaBiosphere Reserve, Gabon. European Journal of Medicinal Plants, 3(2), pp 174-205.

12. Betti, J.-L., Yongo O.D., Obiang Mbomio, D., Midoko Iponga, D. \& Ngoye, A. (2013b). Ethnobotanical and floristical study of Alstonia boonei De Wild. (Apocynaceae) in the Makokou region, Ogoué-Ivindo province, Gabon. International Journal of Agricultural Sciences, 3(3), pp 459-469.

13. Bokatola, M.C. (2013). Gestion de la biodiversité végétale et savoirs endogènes à OYO. Mémoire de C.A.P.E.S., Département des Sciences Naturelles, Ecole Normale Supérieure, Université Marien NGOUABI, Brazzaville (Congo).

14. Bouquet, A. (1967). Inventaire des plantes médicinales et toxiques du Congo. Mémoire ORSTOM, Brazzaville, Paris, (France).

15. Bouquet, A. (1969). Féticheurs et médecines traditionnelles du Congo (Brazzaville). Paris, Mémoire ORSTOM $n^{0} 36$, Brazzaville, Paris (France).

16. Camara, P.E.A.S. (2011). A Review of Taxithelium Subgenus Taxithelium (Bryophyta, Pylaisiadelphaceae). Systematic Botany, 36(4), pp 824-835.

17. CNSEE (2010). Le RGPH-2007 en quelques chiffres. MEPATI, UNFPA, Brazzaville (Congo).

18. Cusset, G. (1989). La flore et la végétation du Mayombe congolais : état des connaissances. In : Sénéchal, J., Makuta Kabala \& Fournier, F. (Eds) Revue de connaissances sur le Mayombe. Unesco, Paris (France).

19. Dalziel, J.M. (1956). Useful plants of West Tropical Africa, Crown Agents for Overseas Government, Londres, Grande Bretagne.

20. Descoings, B. (1975). Les Grandes régions naturelles du Congo, Candollea, 30, pp 90-120.

21. Eilert, U., Wolters, B. \& Mahrstedt, A. (1980). Antibiotic principles of seeds of Moringa oleifera. Planta Medica, 39, 235p. 
22. Farsworth, N.R., Akerele, O., Bingel, A.S., Soejarto, D.D. \& GUO, Z. (1986). Place des plantes médicinales dans la thérapeutique. Bulletin de l'OMS, 64(2), pp 159-175.

23. Frontier, S., Pichod-Viale, D., Leprêtre, A., Davoult, D. \& Luczak, C. (2008). Écosystèmes: Structure, Fonctionnement, Evolution $(4 \mathrm{e}$ édn). Dunod, Paris (France).

24. Gartlan, S. (1989). La Conservation des Écosystèmes forestiers du Cameroun. IUCN, Gland (Switzerland) and Cambridge (UK).

25. Grenand, P. \& Prevost, M. (1994). Les plantes colorantes utilisées en Guyane Française. Journ. d'Agric. Trad. et de Bota. Appl., 36(1), pp 139-172.

26. Grenand, P., Moretti, C. \& Jacquemin, H. (1987). Pharmacopées traditionnelles en Guyane : Créoles, Palikur, Wapayi, coll. Mémoires, 108, ORSTOM, Paris (France).

27. Hakizamungu, E. \& Weri, M. (1988). Usage of medicinal plants in the treatment of malaria in the traditional medicine of Rwanda. Bull. Méd. Trad. Pharm., 2(1), pp 11-17.

28. Hecketsweiler, P. \& Mokoko Ikonga, J. (1991). La réserve de Conkouati : Congo, le secteur Sud-est. UICN, Gland (Suisse).

29. Hecketsweiler, P., Doumenge, C. \& Mokoko Ikonga, J. (1991). Le Parc National d'Odzala, Congo. UICN, Gland (Suisse).

30. Höft, M., Baril, S.K. \& Lykke, A.M. (1999). Quantitative ethnobotany. Applications of multivariate and statistical analyses in ethnobotany. People and plants working paper, UNESCO, 6, pp 1-46.

31. Idowu, O.A., Soniran, O.T., Ajana, O. \& Aworinde, D.O. (2010). Ethnobotanical survey of antimalarial plants used in Ogun State, Southwest Nigeria. Afr. J. Pharm. Pharmacol., 4(2), pp 055-060.

32. Iwu, M.M. (1993). Handbook of African medicinal plants. CRS press, Boca Raton Ann Arbor London (Grande Bretagne) \& Tokyo (Japon).

33. Jain, S.R. \& Sharma, S.N. (1967). Hypoglycaemic drugs of Indian indigenous origin. Planta Medica, 15, pp 429-442.

34. Kerharo, J. (1974). La pharmacopée sénégalaise traditionnelle. Plantes médicinales et toxique. Ed. Vigot, Paris (France).

35. Kimpouni, V. \& Motom, M. (2012). Empirisme et exploitation traditionnelle de la flore par les populations riveraines du lac Cayo (Congo - Brazzaville). Annales de l'Université Marien Ngouabi, 12 13(4), pp 83-100.

36. Kimpouni, V. (1999). A preliminary market survey of non-wood forest products traded in the Pointe-Noire markets (Congo-Brazzaville). In: Sunderland T.C.H., Clark L.E. \& Vantomme P. (eds.) Non Wood Forest Products of Central Africa Current Research issues and 
prospects for conservation and development. CARPE-FAO, Rome (Italie).

37. Kimpouni, V. (2001). Contribution aux études ethnobotaniques et floristiques de la forêt de Lossi (R.P. Congo) : Les plantes de cueillette à usage alimentaire. Systematics and Geography of plants, 71, pp 679686.

38. Kimpouni, V., Apani, E. \& Motom, M. (2011). Plantes ichtyotoxiques et particularisme des usages au Congo (Brazzaville). International Journal of Biological Chemical Sciences, 5(3), pp 979-990.

39. Kimpouni, V. \& Motom, M. (2006). Note sur Inga edulis (Leguminosae, Mimosaceae) au Congo-Brazzaville, In : Notes on tropical African plants. Systematics and Geography of plants, 6, pp 245-250.

40. Kimpouni, V., Koubouana, F., Apani, E., Motom, M. \& MakitaMadzou, J.-P. (2012) Contribution à l'inventaire et à l'utilisation des plantes à effets psychotropes et toxiques au Congo (Brazzaville). Phytothérapie, 10, pp 19-24. Digital Object Identifier (DOI) 10.1007/s10298-012-0684-8, Springer-Verlag France

41. Kimpouni, V. \& Nguembo, J. (2007). Aspects d'exploitation et d'utilisation artisanales des produits forestiers d'origine végétale à Lossi, Congo (Brazzaville). Proceeding 18 ${ }^{\mathrm{e}}$ Congrès AETFAT (27 février au 2 mars, Yaoundé - Cameroun).

42. Kimpouni, V., Mbou, P., Gakosso, G. \& Motom, M. (2013). Biodiversité floristique du sous-bois et régénération naturelle de la forêt de la Patte d'Oie de Brazzaville, Congo. International journal of Biological and Chemical Sciences, 7(3), pp 1255-1270.

43. Lavergne, R. \& Véra, R. (1989). Médecine traditionnelle et pharmacopée: étude ethnobotanique des plantes utilisées dans la pharmacopée traditionnelle à la Réunion.

44. Lebrun, J.-P. \& Stork, L. (1991-1997). Enumération des plantes à fleur d'Afrique tropicale. 4 vols. Genève: Editions des conservatoire et Jardin botaniques de Genève.

45. Makany, L. (1976). Végétation des plateaux teke (Congo). Coll. Travaux Univ. Brazzaville, Brazzaville (Congo).

46. Mamboueni, J.C. (2017). Phytodiversité et pharmacopée traditionnelle de la communauté Kaamba de Madingou (Bouenza - Congo). Mémoire de Master Enseignement, ENS-UMNG, Brazzaville (Congo).

47. Martin, G.J. (1995). Ethnobotany: A manuel methods. Chapman et Hall, London (Grande Bretagne).

48. Martin, G. (1962). Les Sols de la vallée du Niari. Bureau des sols de 1'A.E.F. rapport ORSTOM à diffusion restreinte, Brazzaville (Congo). 
49. Mpassi, P. (2007). Contribution à l'inventaire floristique et ethnobotanique des plantes utiles de Kimbedi. Mémoire C.A.P.E.S., Département des Sciences Naturelles, Ecole Normale Supérieure, Université Marien NGOUABI, Brazzaville (Congo).

50. Nkounkou Loufoukou, R.C. (2012). Savoirs endogènes et conservation de la floriculture urbaine à Brazzaville. Mémoire de C.A.P.E.S., Département des Sciences Naturelles, Ecole Normale Supérieure, Université Marien NGOUABI, Brazzaville (Congo).

51. Nzila, J.D.D. (1992). Etude des Transformations Structurales et Physico-Chimiques d'un sol Ferralitique Acide de la Vallée du Niari (Congo, Soumis à la pratique de l'écobuage). Thèse de Doctorat. Université Paris XII, Paris (France).

52. Nzila, J.D.D. (1996). Caractéristiques des Sols du Congo et Problèmes d'Aménagements des sols. Document U.R. 2PI, Brazzaville (Congo).

53. Olivier, B. (1959). Medecinal Plants in Nigeria. College of Arts, Science and Technology, Ibadan (Nigeria).

54. Peters, C.M. (1997). Sustainable exploitation of non-wood forest products in tropical rain forests: ecology hand book. General sery of the biodiversity program, $\mathrm{n}^{\circ} 2$. WWF-NC-WRI/USAID, Washington 'USA).

55. Profizi, J.-P., Makita-Madzou, J.-P., Milandou, J.-C., Karanda, C.N., Motom, M. \& Bitsindou, I. (1993). Ressources végétales non ligneuses des forêts du Congo. Plan d'Action Forestier Tropical du Congo (PAFT-Congo). Univ. Marien Ngouabi, Brazzaville (Congo).

56. Puig, H. (2001). La Forêt Tropicale Humide. Belin, Paris (France).

57. Ramachandran, C., Peter, K.V. \& Gopalakrishan, P.K. (1980). Drumstik (Moringa Oleifera). A multi-purpose Indian vegetable. Economic Botany, 34(3), pp 276-282.

58. Raponda-Walker, A. \& Sillans, R. (1961). Les plantes utiles du Gabon. éd. Lechevalier, Paris (France).

59. Rivera, G. (1941). Preliminary chemical and pharmacological studies on cundeamor Momordica charantia L. American journal of Pharmacy, 113, pp 282-297.

60. Saluja, M.P., Kapil, R.S. \& Popli, S.P. (1978). Studies on medicinal plants : part VI. Chemical constituents of Moringa Oleifera Lamk. (hybrid variety) and isolation of 4-hydroxymellien. Indian journal of chemistry, section B, 16B(11), pp 1044-1045.

61. Samba-Kimbata, M.-J. (1978). Le climat Bas congolais. Thèse de doctorat, Université de Dijon, Dijon, Dijon (France).

62. Saotoing, P., VroumsiaToua, Tchobsala, Tchuenguem Fohouo, F.N., Njan Nloga, A.M. \& Messi, J. (2011). Medicinal plants used in traditional treatment of malaria in Cameroon. 
63. Slayback, D. (2003). Landcover change in the Takamanda Forest reserve, Cameroon: 1986-2000, pp. 173-179, in: Comiskey, J.A., Sunderland, T.C.H. \& Sunderland-Groves, J.L. (Eds) Takamanda : the biodiversity of an African rainforest. SI/MAB (USA).

64. Sofowara, A. (1996). Plantes médicinales et médecine traditionnelle d'Afrique, Ed. Karthala, Paris (France).

65. Tailfer, Y. (1989). La forêt dense de l'Afrique centrale : Identification pratique des principaux arbres. Volume 1 et 2, Paris : ACCT et CTA, Paris (France).

66. Ticktin, T. (2004). The ecological implications of harvesting nontimber forest products. J. Appl. Ecol., 41(1), pp 11-21.

67. Titanji, V.P.K., Zofou, D., Ngemeneya, M.N. (2008). The Antimalarial potential of medicinal plants used for the treatment of malaria in Cameroonian Folk Medicine. Afr. J. Trad. Cam., 5(3), pp 302-321. Tropical Diseases Web Ring (2000). Questions/Réponses sur le paludisme. Malaria FAQ. http: //myweb.worldnet.net/ ditjaste/faqmalfr.html).

68. Trochain, J.-L. (1980). Ecologie Végétale de la zone intertropicale non désertique. Université de Toulouse, Toulouse (France).

69. UICN (1996). L'Atlas pour la conservation des forêts tropicales d'Afrique. UICN, Gland (Suisse).

70. Vennetier, P. (1977). Atlas de la République Populaire du Congo. Edition Jeune Afrique, Paris (France).

71. Verpoorte R. (1999). pharmacognosy in new millennium leadfinding and biotechnology. J. Pharm. Pharmacol., 52, pp 253-262. DOI: 10.1211/0022357001773931

72. Watt, J. \& Breyer-Brandwijk, M.G. (1962). The Medicinal and Poisonous Plants of South and Eastern Africa, E.\&S. Livingstone, Edimbourg.

73. Wezel, A. (2002). Plantes médicinales et leur utilisation traditionnelle chez les paysans au Niger. Etudes Flor. Vég. Burkina Faso, 6, pp 9-18. 